\title{
Baryonic Resonances from the Interactions of the Baryon Decuplet and Meson Octet
}

\author{
E. Oset, S. Sarkar and M. J. Vicente Vacas \\ October 3, 2018 \\ Departmento de Física Teórica and IFIC, Centro Mixto Universidad de Valencia-CSIC, \\ Institutos de Investigación de Paterna, Aptd. 22085, 46071 Valencia, Spain
}

\begin{abstract}
We study $S$-wave interactions of the baryon decuplet with the octet of pseudoscalar mesons using the lowest order chiral Lagrangian. We find two bound states in the $S U(3)$ limit corresponding to the octet and decuplet representations. These are found to split into eight different trajectories in the complex plane when the $S U(3)$ symmetry is broken gradually. Finally, we are able to provide a reasonable description for a good number of 4-star $\frac{3}{2}^{-}$resonances listed by the Particle Data Group. In particular, the $\Xi(1820)$, the $\Lambda(1520)$ and the $\Sigma(1670)$ states are well reproduced. We predict a few other resonances and also evaluate the couplings of the observed resonances to the various channels from the residues at the poles of the scattering matrix from where partial decay widths into different channels can be evaluated.
\end{abstract}

\section{Introduction}

The introduction of unitary techniques in a chiral dynamical treatment of the meson baryon interaction has been very successful. It has lead to good reproduction of meson baryon data with a minimum amount of free parameters, and has led to the dynamical generation of many 
low lying resonances which qualify as quasibound meson baryon states [1, 2, 3]. In particular, the application of these techniques to the $s$-wave scattering of the baryon octet and the pseudoscalar meson octet have led to the successful description of many $J^{P}=\frac{1}{2}^{-}$resonances like the $N^{*}(1535)$, the $\Lambda(1405)$, the $\Lambda(1670)$, the $\Sigma(1620)$ and the $\Xi(1620)$ [4, [5, 6, 7, 8]. Naively one may expect that this scheme is not suitable for studying $d$-wave resonances due to a large number of unknown parameters in the corresponding chiral Lagrangian. However $d$-wave resonances could be studied in $s$ wave interactions of the meson octet with the baryon decuplet [9], in which case chiral dynamics is quite predictive. The fact that some well known $d$ wave resonances like the $N^{*}(1520), N^{*}(1700), \Delta(1700)$ have very large branching ratios to the $N \pi \pi$ channel though the $N \pi$ channel is favoured by phase space, lends support to this scheme.

\section{Baryon Decuplet-Meson Octet Interaction: Re- sults and Discussion}

We have performed a systematic study [10] of the $s$-wave interaction of the baryon decuplet with the meson octet taking the dominant lowest order chiral Lagrangian, which accounts for the Weinberg Tomozawa term. In this talk we will present the main results of this work.

The tree-level scattering amplitude involving the baryon decuplet and the pseudoscalar octet is used as the kernel of the Bethe Salpeter equation to obtain the transition matrix fulfilling exact unitarity in coupled channels. In this approach, the only free parameter is the subtraction constant in the dimensionally regularized meson baryon loop function for

which we took a natural size value. We have looked in detail at the $\frac{3}{2}^{-}$resonances which are generated dynamically by this interaction, by searching for poles of the transition matrix in the complex plane in different Riemann sheets. The search was done systematically, starting from an $S U(3)$ symmetric situation where the masses of the baryons are made equal and the same is done with the masses of the mesons. In this case we found attraction in an octet, a decuplet and the 27 representation, while the interaction was repulsive in the 35 representation. In the $S U(3)$ symmetric case all states of the $S U(3)$ multiplet are degenerate and the resonances appear as bound states with no width. As we gradually break $S U(3)$ symmetry by changing the masses, the degeneracy is broken and the states with different 

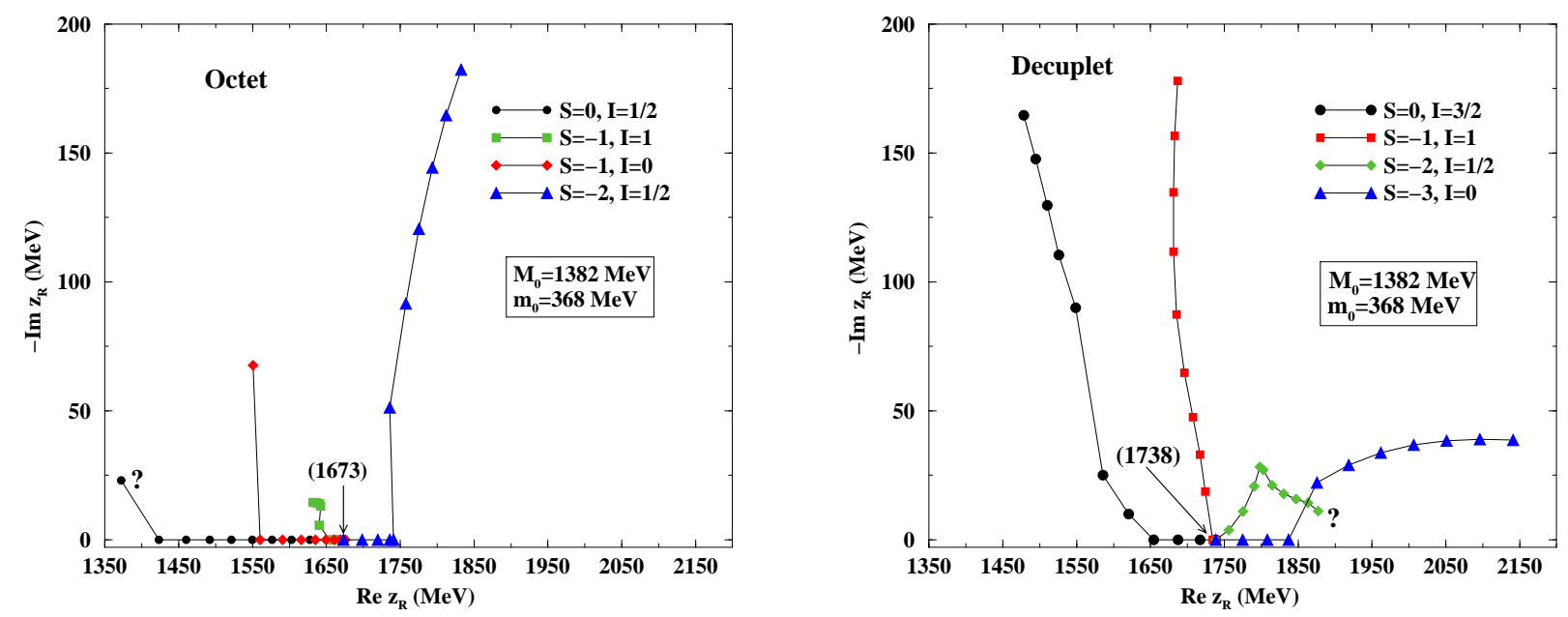

Figure 1: Trajectories of the poles in the scattering amplitudes for different values of the $S U(3)$ breaking parameter.

strangeness and isospin split apart generating pole trajectories in the complex plane which lead us to the physical situation in the final point, as seen in fig. 1. This systematic search allows us to trace the poles to their $S U(3)$ symmetric origin, although there is a mixing of representations when the symmetry is broken. In addition we also find poles which only appear for a certain amount of symmetry breaking and thus have no analog in the symmetric case.

The search for poles is an ideal technique to identify resonances in addition to visual inspection of the amplitudes. On the other hand, in ref. [9] the speed plot technique is used to identify resonances. However, the speed plot, involving derivatives of the amplitudes, produces artificial peaks at the thresholds of new channels, because the cusp of the amplitudes, and should not be used there. Neglecting this fact, some peaks which are actually reflections of cusps in the speed plot are misidentified as resonances in [9] while we do not find poles, not even making reasonable changes in the input data.

We have also evaluated the residues of the poles from where the couplings of the resonances to the different coupled channels were found and this allowed us to make predictions for partial decay widths into a decuplet baryon and a meson. There is very limited experimental information on these decay channels but, even then, it represents an extra check of consistency of the results which allowed us to more easily identify the resonances found with some resonances known, or state that the resonance should correspond to a new resonance not yet 

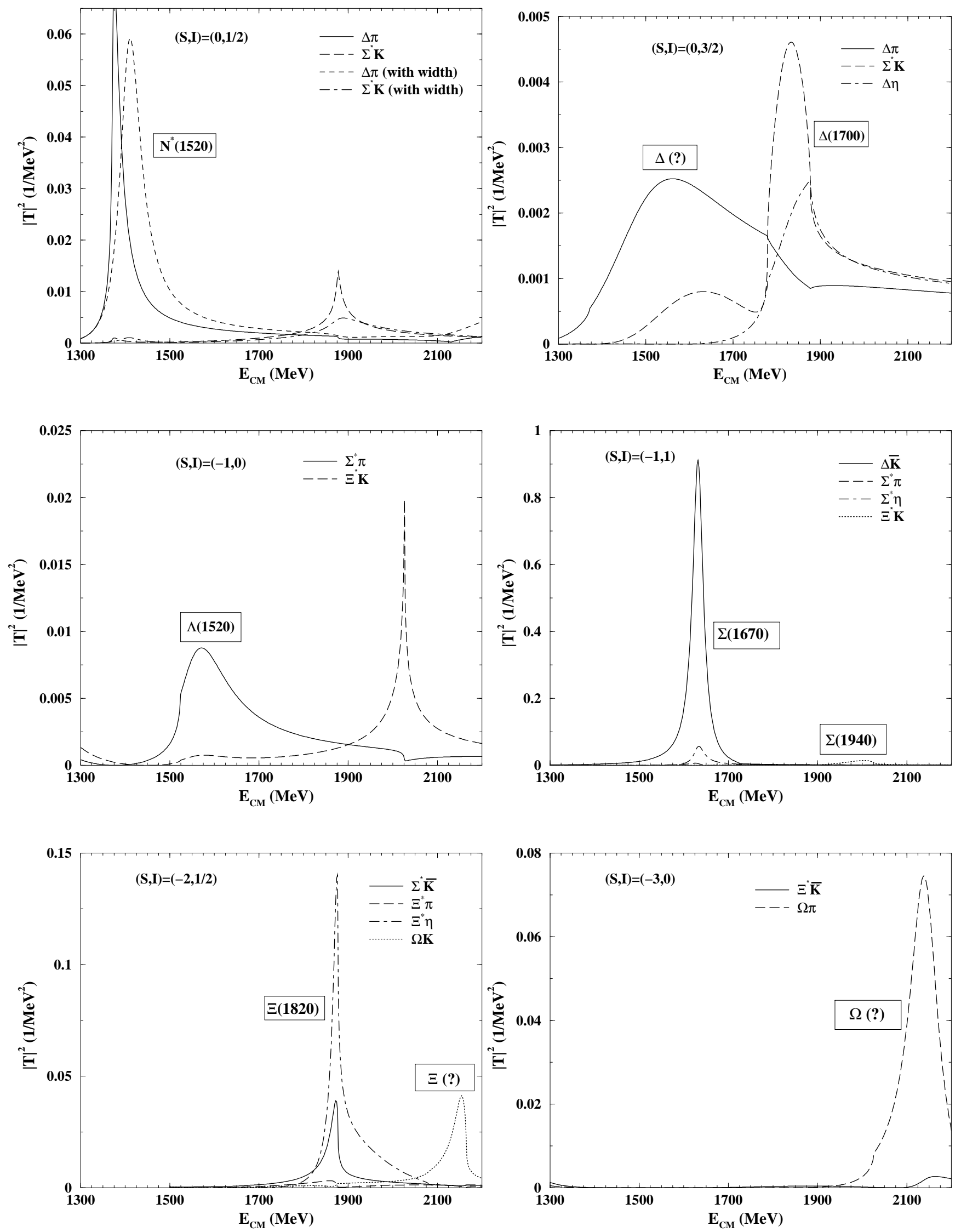

Figure 2: Amplitudes for $3 / 2^{-}$resonances generated dynamically in decuplet baryon - pseudoscalar octet interactions. 
reported in the Particle Data Book (PDB). In particular, in view of the information of the pole positions and couplings to channels we could associate some of the resonances found to the $N^{*}(1520), \Delta(1700), \Lambda(1520), \Sigma(1670), \Sigma(1940), \Xi(1820)$ resonances tabulated in the PDB. We could also favour the correspondence of a resonance found in $S=-3, I=0$ to the $\Omega(2250)$ on the basis of the quantum numbers, position and compatibility of the partial decay width found with the total width of the $\Omega(2250)$. The scattering amplitudes for different values of strangeness and isospin as a function of the C.M. energy are shown in fig. 2]

We also found several extra resonances, well identified by poles in the complex plane which do not have a correspondent one in the PDB. Some of them are too broad, which could justify the difficulty in their observation, but two other resonances, the $\Delta(1500)$ with a width around $300 \mathrm{MeV}$ and the $\Xi(2160)$ with a width of about $40 \mathrm{MeV}$ stand much better chances of observation. The first one because of its large strength in the $\Delta \pi$ channel and the second one because of its narrowness.

In addition, our study produces couplings of the resonances to baryon-meson channels which could facilitate the identification when further information on these branching ratios is available. We have found that in some cases the dynamically generated resonances couple very strongly to some channels with the threshold above the resonance energy, which makes them qualify as approximately single channel quasibound meson-baryon states.

Lastly, we mention the very interesting case concerning the $\Delta K$ resonance [11] found in $S=1, I=1$ which shows up as a pole in the unphysical Riemann sheet and leads to $\Delta K$ cross sections much larger than the corresponding $\Delta K$ cross sections in $I=2$. We suggest that these two cross sections could be seen in $\Delta K$ production in $p p \rightarrow \Lambda \Delta K$ and $p p \rightarrow \Sigma \Delta K$ reactions and could provide evidence for this new 'exotic pentaquark' state, although its structure is more efficiently taken into account in the meson baryon picture where it has been generated. This state, however, appears as a broad bump above the $K \Delta$ threshold and its strength is also tied to details of the interaction. Hence, the ultimate fate of this resonance is difficult to predict at the present stage.

Acknowledgments: This work is partly supported by DGICYT contracts BFM200201868, and the E.U. EURIDICE network contract HPRN-CT-2002-00311. This research is part of the EU Integrated Infrastructure Initiative Hadron Physics Project under contract 
number RII3-CT-2004-506078.

\section{References}

[1] N. Kaiser, P. B. Siegel and W. Weise, Nucl. Phys. A 594 (1995) 325; N. Kaiser, T. Waas and W. Weise, Nucl. Phys. A 612 (1997) 297.

[2] E. Oset and A. Ramos, Nucl. Phys. A 635 (1998) 99.

[3] J. A. Oller and U.-G. Meißner, Phys. Lett. B 500 (2001) 263.

[4] T. Inoue, E. Oset and M. J. Vicente Vacas, Phys. Rev. C 65 (2002) 035204.

[5] D. Jido, J. A. Oller, E. Oset, A. Ramos and U. G. Meissner, Nucl. Phys. A 725 (2003) 181.

[6] C. Garcia-Recio, J. Nieves, E. Ruiz Arriola and M. J. Vicente Vacas, Phys. Rev. D 67 (2003) 076009 arXiv:hep-ph/0210311.

[7] C. Garcia-Recio, M. F. M. Lutz and J. Nieves, Phys. Lett. B 582 (2004) 49.

[8] E. Oset, A. Ramos and C. Bennhold, Phys. Lett. B 527 (2002) 99 [Erratum-ibid. B 530 (2002) 260];A. Ramos, E. Oset and C. Bennhold, Phys. Rev. Lett. 89 (2002) 252001.

[9] E. E. Kolomeitsev and M. F. M. Lutz, Phys. Lett. B 585 (2004) 243.

[10] S. Sarkar, E. Oset and M. J. Vicente Vacas, Nucl. Phys. A 750 (2005) 294 arXiv:nucl-th/0407025.

[11] S. Sarkar, E. Oset and M. J. Vicente Vacas, Eur. Phys. J. A 24 (2005) 287 arXiv:nucl-th/0404023. 